\title{
The Evolution of Laparoscopic Surgery in the Field of Morbid Obesity
}

\author{
Rudolf A. Weiner \\ Zentrum für Minimal-Invasive Chirurgie, Chirurgische Klinik, Krankenhaus Sachsenhausen, Frankfurt/M., Germany
}

There is a world epidemic of overweight, obesity, and morbid obesity, encompassing 1.7 billion people. At the 3rd Frankfurt Meeting for Laparoscopic Surgery of Morbid Obesity, held at November 11-12, 2004, surgeons from 28 countries were registered. They were from Brazil, Mexico, USA, and nearly all European countries. For the first time guests from Iran, Kuwait and other countries in the Near East attended at the meeting. The great interest of surgeons from different parts of the world demonstrates the importance of the worldwide obesity problem.

We would like to thank the head of the organizing committee, Sylvia Weiner, Obesity Academy Frankfurt e. V., for the excellent work. The 4th Frankfurt Meeting for Laparoscopic Obesity Surgery is scheduled for November 2006 ( $w w w$.frankfurter-meeting.de) and will be organized again by the Obesity Academy Frankfurt e. V.

The prevalence of obesity in the USA is increasing to epidemic proportions. Currently, more than $60 \%$ of Americans and $51 \%$ of Germans are overweight. In 2003 more than 9 millions in Germany were obese (body mass index $(\mathrm{BMI})>30 \mathrm{~kg} / \mathrm{m}^{2}$ ). Obesity has been identified as a major risk factor for many diseases, including cardiovascular disease, cancer, and type II diabetes. The cost associated with treatment of obesity are similarly extreme. In the USA, more than USD 30 billion per year is spent on dietary and behavioral treatments for obesity. A study from USA reported that healthcare cost are $44 \%$ greater among severely obese patients. Whereas the problem of obesity is an international one, the effects are especially acute in the USA where the cost of treating obesity and related ailments has been estimated to account for $7 \%$ of the national healthcare budget. In Germany no data were available until now. The last calculations were done in 1993.

Whereas a variety of medications are available for treatment of obesity, none results in a long-term loss of more than $10 \%$ of body weight. The current standard for treatment of severe obesity, defined as a BMI of greater than $35 \mathrm{~kg} / \mathrm{m}^{2}$ with co-morbidities and generally greater than $40 \mathrm{~kg} / \mathrm{m}^{2}$, is surgical. Bariatric surgery today is the only effective therapy for morbid obesity.

The large, but non-randomized Swedish Obese Subjects (SOS) study compared different types of obesity surgery versus conserva- tive treatment in a matched-pair design. Women and men with a BMI greater than 38 and $34 \mathrm{~kg} / \mathrm{m}^{2}$, respectively, were studied over 2 years. They lost significantly more weight after surgical than after non-surgical treatment, and this weight loss resulted within 2 years in significant improvements of co-morbidities such as diabetes (from 19 to $10 \%$ ), hypertension (from 53 to $31 \%$ ), sleep apnea (from 23 to $8 \%$ ), dyspnea when climbing stairs (from 87 to 19\%), and chest pain when climbing stairs (from 28 to $4 \%$ ). The SOS study also reported health-related quality of life (QoL) to be directly correlated with weight loss. As there was a significant difference in QoL even between women with $30-40 \mathrm{~kg}$ weight loss and those with more than $40 \mathrm{~kg}$ weight loss, it seems as if obesity surgery should aim at the largest possible loss of excess weight. Prescription cost for treatment of co-morbidities associated with morbid obesity are a considerable annual healthcare expenditure. Obesity surgery can decrease the prescription medication requirements, resulting in significant cost savings in the treatment of obesity-related hypertension and diabetes. Several studies found a $77 \%$ reduction in total cost of diabetic and antihypertensive medications.

Currently, a number of different surgical procedures are available for treatment of the severely obese patient. These procedures generate weight loss from two mechanisms of action: restriction and malabsorption. Essential is that patients are well informed regarding the specific changes in lifestyle, eating habits, and bowel habits that may accompany the procedure being suggested for them because the different operations entail substantially different lifestyle modifications. Given the enormous importance of morbid obesity, surgical therapies have become increasingly popular. The number of procedures performed has more than doubled within a few years. This dramatic growth also can be attributed in part to the introduction of new surgical techniques, e.g. the adjustable silicone gastric band (ASGB), and the rise of laparoscopic surgery. Traditionally, the two types of operations for morbid obesity are gastric restrictive operations (where food intake is restricted) and malabsorptive operations (where food is diverted from absorption by way of a gastrointestinal shortcut).

All types of obesity surgery may be performed using laparoscopic

\section{KARGER}

Fax +497614520714

(c) 2005 S. Karger GmbH, Freiburg

E-mail Information@Karger.de

www.karger.com
Accessible online at: www.karger.com/cga
Prof. Dr. med. Rudolf A. Weiner

Chirurgische Klinik, Krankenhaus Sachsenhausen

Schulstraße 31, 60954 Frankfurt, Germany

Tel. +49 $696605-1199$, Fax -15 10

E-mail rweiner@khs-ffm.de 
surgical techniques to minimize perioperative morbidity and postoperative recovery time. In order to optimize the outcome of this type of procedure, bariatric surgery should be performed on carefully selected patients, in bariatric centers specially equipped to care for the obese, and within a broadly based, multidisciplinary setting that provides lifelong postoperative care. As obesity surgery has various competing aims such as weight loss, adjustability, reversibility, and safety, it is difficult to draw universally valid conclusions regarding the optimal bariatric procedure. For all types of surgery, overwhelming evidence from case series exists on safety, efficacy, and effectiveness, but much less data are available on the comparative evaluation of different bariatric procedures. Therefore, the decision must be made with the patient's individual situation and surgeon's expertise in mind. A profound knowledge of the different malabsorptive and gastric restrictive procedures and their pathophysiologic consequences is indispensable.

The number of bariatric procedures is increasing worldwide. The highest incidences are seen in America, Europe and the Near East, followed by Australia. An analysis by Henry Buchwald, president of the International Federation for Surgery of Obesity (IFSO) in 2004 has shown:

- In the years 2002-2003, 146,301 bariatric surgery operations were performed by 2,839 bariatric surgeons; 103,000 of these operations were performed in the USA/Canada by 850 surgeons.

- The earliest start date for bariatric surgery was 1953 in the USA; IFSO was founded in 1995. In the years 2002-2003, 37.15\% of operations were open and $62.85 \%$ laparoscopic.
- The 6 most popular procedures by weighted averages were: laparoscopic gastric bypass (25.7\%), laparoscopic ASGB (24.1\%), open gastric bypass (23.1\%), laparoscopic long-limb gastric bypass $(8.9 \%)$, open long-limb gastric bypass $(7.5 \%)$, and open vertical banded gastroplasty $(4.2 \%)$.

- Pooling open and laparoscopic procedures, relative percentages were: gastric bypass $66 \%$, ASGB $24 \%$, vertical banded gastroplasty $5 \%$, and biliopancreatic diversion/duodenal switch $5 \%$.

- Categorizing into restrictive/malabsorptive, purely restrictive, and primarily malabsorptive, the relative distribution of procedures was 65,30 , and $5 \%$, respectively.

- ASGB was performed in 23 (95\%), gastric bypass in $21(88 \%)$, vertical banded gastroplasty in $19(79 \%)$, and biliopancreatic diversion/duodenal switch in $16(67 \%)$ countries. Purely restrictive procedures were performed in 24 (100\%), restrictive/malabsorptive procedures in $21(88 \%)$, and primarily malabsorptive procedures in $18(75 \%)$ countries.

- Bariatric surgery is expanding exponentially to meet the global epidemic of morbid obesity. Operative procedures in bariatric surgery are in flux, and specific geographic trends and shifts are evident. Yet, of the patients qualifying for surgery, only about $1 \%$ are receiving this therapy - the only effective treatment currently available.

A selected number of presentations of the 3rd Frankfurt Meeting will be published in this supplement of Chirurgische GastroenterOLOGIE INTERDISZIPLINÄR. 